\title{
Effect of Camera Tipping on the Location of the Principal Point ${ }^{1,2}$
}

\author{
Francis E. Washer
}

\begin{abstract}
Asymmetric distortion is introduced in a lens-camera combination when the camera is incorrectly alined for calibration. A method is described whereby the magnitude and direction of the angle of camera tipping can be determined from analysis of the asymmetric values of distortion for the case of the object lying at equal but opposite angles from the central line of sight. The displacement of the central image from the principal point of autocollimation is then readily determined. Theoretical analysis of the problem is given and the results confirmed by experiment. The point of symmetry and the principal point of autocollimation coincide for the case of no prism effect present on the lens.
\end{abstract}

\section{Introduction}

One of the problems that arises during the calibration of precision aerial-mapping cameras is the accurate location of the proper principal point [1] ${ }^{3}$ with respect to the center of collimation (the point determined by the intersection of lines joining opposite pairs of collimation index markers). The principal point in photogrammetry is defined as the point at the foot of the perpendicular drawn from the interior perspective center to the plane of the photograph. The proper manner of locating this point has long been a subject of discussion. There are at present three different procedures that are employed in locating a point which is believed to be a sufficiently close approximation to the true principal point.

The first method, which has enjoyed considerable popularity, locates a point called the principal point of autocollimation $[2,3]$, which is the center of the image formed in the emulsion plane by the camera lens from an incident beam of parallel light, which, in the object space, is perpendicular to the emulsion plane. This point has usually been designated "center cross" [4] by the writer for convenience. This point is located by the simplest of the three methods, and its popularity undoubtedly derives from this simplicity. To locate it, one needs only to aim an autocollimating telescope at a distant object. The camera is then interposed between the object and the telescope; a plane parallel plate with a mirror surface is pressed against the focal plane. With the aid of the autocollimating telescope, one can easily adjust the camera until its focal plane is normal to the line of sight of the telescope. The reflecting plate is then replaced by a photographic plate and the distant object is photographed. If the collimation index markers are simultaneously photographed, one can then easily locate the center cross with respect to the center of collimation.

The second method, which was developed at the Bureau, is based on the knowledge that most lenses

1 This is the second paper of a series dealing with problems that relate to the calibration of precision airplane mapping cameras (see reference [13] at the end of this paper).

2 This work was sponsored in part by the U. S. Air Force.

3 Figures in brackets indicate the literature references at the end of this paper. suffer from small errors in centering that cause the lens to behave (in a first approximation) as though it were composed of a well-centered lens plus a thin prism $[4,5]$. This is further accentuated in the calibration of precision cameras by the presence in front of the lens of a filter that is seldom truly plane parallel, which accordingly introduces a true prism effect. This prism effect causes a displacement of the center cross from the position it would have occupied in the absence of prism effect. This immediately poses the question as to which of these locations should properly be regarded as the principal point. It has been the custom at the Bureau to regard the point that would normally have been occupied by the center cross in the absence of prism effect as the proper principal point of the photograph. It is an invariant point when located under standard conditions of locating the center cross and is not affected by subsequent changes in filters having different degrees of surface parallelism. The actual shift of the principal point from the center cross is easily determined from analysis of the asymmetric distortion pattern produced by such prism effect.

The third method came out of attempts to calibrate cameras by a field method [6]. It was not customary nor readily practicable to aim the camera at the central target of a given target-array with the aid of an autocollimating telescope and, consequently, small errors of alinement sometimes were present. Such errors of alinement, called camera tipping, can produce an asymmetric distortion pattern similar but not identical to that produced by prism effect. In the analysis of the measurements made under these conditions of test, it was found possible to make the distortion pattern symmetrical by appropriate adjustment of the angles and image separations from the central image by computable increments. The point about which the distortion is tolerably symmetrical is called the point of symmetry.

It is evident that for an ideal lens-camera combination, where no tipping of the camera occurs and where there is no prism effect in the lens, all three methods will lead to the same result. In other words, for this case, the principal point of autocollimation (or center cross), the principal point, and the point of 
symmetry will be identical. In the present paper, the effect of camera tipping on the displacement of the central image from the original position of the center cross is considered. The magnitude of the asymmetric distortion introduced by such tipping is evaluated theoretically and confirmed experimentally. A method of determining the magnitude of the angle of camera tipping is shown for the case of equal opposite angles. The identity of the principal point of autocollimation and the point of symmetry is shown for the case of a lens having no prism. effect.

\section{Effect of Improper Alinement of Camera for Test}

When the camera is being calibrated under conditions such that the focal plane of the camera is not normal to the line drawn to it from the central target, the calibration data are likely to show the presence of asymmetric distortion [7, 8, 9]. This asymmetric distortion is sometimes confused with the asymmetric distortion produced by prism. effect in the lens $[4,10]$. It is, however, much simpler to eliminate the asymmetry of the distortion values arising from camera tipping than it is to eliminate or reduce the asymmetrical distortion arising from prism effect. In this section, the effect of camera tipping upon the values of focal length and distortion is considered.

\subsection{Determination of the Equivalent Focal Length}

In figure $1, N$ is the rear nodal point of an ideal lens, $L ; O$ is the point where a perpendicular dropped from $N$ intersects the focal plane, consequently $\mathrm{NO}$ is equal to $f$, the equivalent focal length. The points of intersection with the focal plane of rays

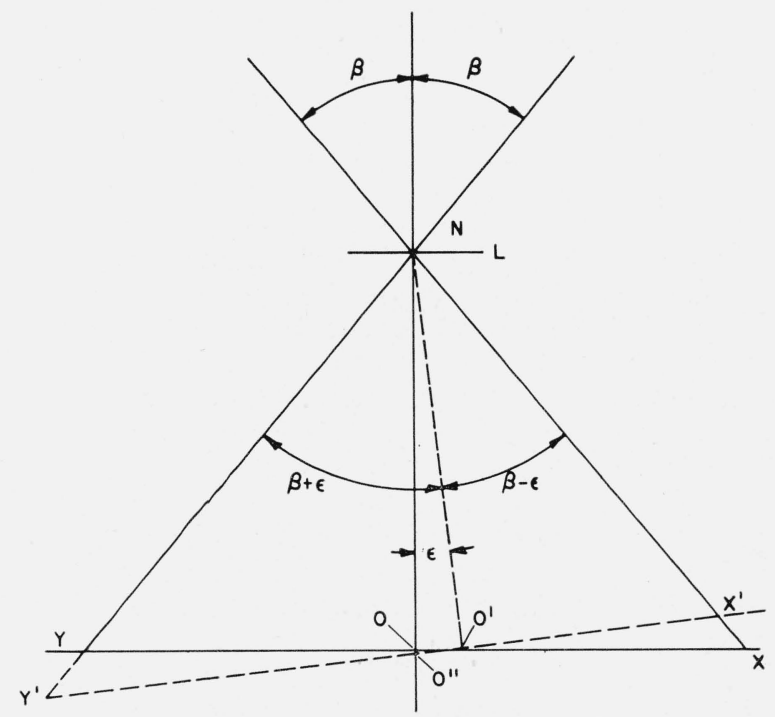

Figure 1. Schematic drawing showing the image shift produced by rotating the camera by amount $\epsilon$ about an axis normal to the plane of the paper and passing through the rear nodal point $N$ of the lens $L$. inclined at angles $\beta$ with the optical axis are designated $X$ and $Y$. As $L$ is an ideal lens having no distortion,

$$
O X=O Y=f \tan \beta .
$$

Let the camera be rotated by amount $\epsilon$ about a line through $N$ normal to the plane of the paper. The foot of the perpendicular from $N$ moves to $O^{\prime}$, the image of the central ray is at $O^{\prime \prime}$, and the images formerly found at $X$ and $Y$ are at $X^{\prime}$ and $Y^{\prime}$. The relations connecting the various quantities and distances are

and

$$
\begin{aligned}
& O^{\prime} O^{\prime \prime}=f \tan \epsilon \\
& O^{\prime \prime} X^{\prime}=f \tan (\beta-\epsilon)+f \tan \epsilon
\end{aligned}
$$

$$
O^{\prime \prime} Y^{\prime}=f \tan (\beta+\epsilon)-f \tan \epsilon \text {. }
$$

The distances $O^{\prime \prime} X^{\prime}$ and $O^{\prime \prime} Y^{\prime}$ are the only quantities directly measurable. It is clear from figure 1 that

$$
O^{\prime \prime} X^{\prime}+O^{\prime \prime} Y^{\prime}=f \tan (\beta-\epsilon)+f \tan (\beta+\epsilon)
$$

or

$$
O^{\prime \prime} X^{\prime}+O^{\prime \prime} Y^{\prime}=\frac{2 f \tan \beta}{\cos ^{2} \epsilon\left(1-\tan ^{2} \beta \tan ^{2} \epsilon\right)},
$$

whence

$$
f=\frac{O^{\prime \prime} X^{\prime}+O^{\prime \prime} Y^{\prime}}{2 \tan \beta} \cos ^{2} \epsilon\left(1-\tan ^{2} \beta \tan ^{2} \epsilon\right) .
$$

For small $\epsilon$, the term $\left(1-\epsilon^{2}\right)$ can be substituted for $\cos ^{2} \epsilon$, and $\epsilon^{2}$ for $\tan ^{2} \epsilon$. By neglecting the term in $\epsilon^{4}$, eq (7) becomes

$$
f=\frac{O^{\prime \prime} X^{\prime}+O^{\prime \prime} Y^{\prime}}{2 \tan \beta}\left[1-\epsilon^{2}\left(1+\tan ^{2} \beta\right)\right] .
$$

For very small values of $\epsilon$, the term in $\epsilon^{2}$ can be dropped, and the expression becomes simply

$$
f=\frac{O^{\prime \prime} X^{\prime}+O^{\prime \prime} Y^{\prime}}{2 \tan \beta} \text {. }
$$

Thus, the equivalent focal length, $f$, can be determined to a good degree of approximation by using the measured values of $O^{\prime \prime} X^{\prime}, O^{\prime \prime} Y^{\prime}$, and the known value of $\beta$. The value of $f$, so determined, will be correct to the nearest thousandth of a millimeter for $\epsilon \leq 10 \mathrm{~min}$ for values of the focal length of the order of $150 \mathrm{~mm}$. If eq (9) is used when $\epsilon>10 \mathrm{~min}$, the values of $f$ will be higher than the true value, the error running as high as $0.012 \mathrm{~mm}$ for $\epsilon=30 \mathrm{~min}$. In such case it is necessary to determine $\epsilon$ by the method shown in a later portion of this paper and to use this value of $\epsilon$ in eq (8). The value of $f$ determined with eq (8) agrees with the true value for values of $\epsilon$ as high as $30 \mathrm{~min}$. 


\subsection{Asymmetric Distortion Produced by Improper Alinement}

When a camera is tipped in the manner described in section 2.1, it is found that an asymmetric distortion is introduced by such tipping. This distortion affects the relative location in the image plane even as does the distortion inherent in the lens arising from lens aberrations. There is, however, one pronounced difference. Whereas distortion arising from lens aberrations is symmetric about the optical axis, distortion arising from camera tipping is decidedly asymmetric.

In this section, the magnitude of this asymmetric distortion will be derived in terms of $\beta$ and $\epsilon$ for the case of a lens that is distortion-free as far as lens aberrations are concerned. By referring to figure 1, when distortion is computed from the measurable quantities in the usual manner, the distortion, $D_{1}$, on one side is

$$
D_{1}=O^{\prime \prime} X^{\prime}-O X
$$

or

$$
D_{1}=f[\tan (\beta-\epsilon)+\tan \epsilon-\tan \beta],
$$

which may be written

$$
D_{1}=f \tan \beta \tan \epsilon \frac{(\tan \epsilon-\tan \beta)}{1+\tan \beta \tan \epsilon},
$$

which for small values of $\epsilon$ is equivalent to

$$
D_{1}=f \epsilon \tan \beta\left[\epsilon\left(1+\tan ^{2} \beta\right)-\tan \beta\left(1+\epsilon^{2}\right)\right],
$$

or on neglecting the term in $\epsilon^{3}$,

$$
D_{1}=-f \epsilon \tan ^{2} \beta+\frac{f \epsilon^{2} \tan \beta}{\cos ^{2} \beta} .
$$

The distortion, $D_{2}$, for the other side is

$$
D_{2}=O^{\prime \prime} Y^{\prime}-O Y
$$

or

$$
D_{2}=f[\tan (\beta+\epsilon)-\tan \epsilon-\tan \beta],
$$

which for small values of $\epsilon$ can be shown to be

$$
D_{2}=f \epsilon \tan ^{2} \beta+\frac{f \epsilon^{2} \tan \beta}{\cos ^{2} \beta} .
$$

On inspection of the equations for $D_{1}$ and $D_{2}$, it is obvious that for small $\epsilon$ the second term containing $\epsilon^{2}$ will be very small compared to the first term in both equations. It is, therefore, clear that $D_{1}$ is approximately equal to $D_{2}$ in magnitude but is opposite in sign. The effect of camera tipping is, accordingly, an introduction of a symmetric distortion whose magnitude is a function of $\beta$ and $\epsilon$. It is worthy of mention that, when one is analyzing the results of measurement made on a camera whose direction of tipping is unknown, the sign of the distortion values can serve as a guide in determining the angle of tipping and the resultant direction of displacement of the central image from the true position of the center cross or point of symmetry. The point of symmetry or true position of the center cross lies on the same side of the central image as those points showing maximum negative distortion. To express it another way, the central image is displaced from the center cross toward those points showing maximum positive distortion. To illustrate the effect of camera tipping, computed values of $D_{1}$ and $D_{2}$ are listed in table 1 for the case of a

\begin{tabular}{|c|c|c|c|c|}
\hline \multirow{3}{*}{$\beta$} & \multicolumn{4}{|c|}{ Distortion } \\
\hline & \multicolumn{2}{|c|}{$\begin{array}{c}\text { Average asymmetric } \\
\text { distortion }\end{array}$} & \multicolumn{2}{|c|}{$\begin{array}{l}\text { Resultant average distortion } \\
\text { in image }\end{array}$} \\
\hline & $D_{1}$ & $D_{2}$ & $\frac{D_{2}-D_{1}}{2}$ & $\frac{D_{2}+D_{1}}{2}$ \\
\hline $\begin{array}{l}\text { deg } \\
7.5 \\
15 \\
22.5 \\
30 \\
37.5 \\
45\end{array}$ & $\begin{array}{c}m m \\
-0.014 \\
-.061 \\
-.147 \\
-.287 \\
-.507 \\
-.863\end{array}$ & $\begin{array}{c}m m \\
0.016 \\
.064 \\
.152 \\
.295 \\
.520 \\
.883\end{array}$ & $\begin{array}{c}m m \\
0.015 \\
.063 \\
.150 \\
.291 \\
.514 \\
.873\end{array}$ & $\begin{array}{c}m m \\
0.001 \\
.002 \\
.002 \\
.004 \\
.006 \\
.010\end{array}$ \\
\hline
\end{tabular}
camera tipped through an angle $\epsilon=20 \mathrm{~min}$. Even for this relatively large amount of tip, it can be seen that $D_{1}$ is nearly equal to $D_{2}$ but is opposite in sign.

TABLe 1. Asymmetric distortion, $D_{1}$ and $D_{2}$, induced in the image plane by tipping a camera, equipped with a lens having an equivalent focal length of $150 \mathrm{~mm}$, through an angle $\epsilon=20 \mathrm{~min}$

a. Evaluation of the Distortion

The values of the distortion assigned to a cameralens combination are usually obtained by averaging the values measured at the two corresponding opposite angular separations from the axis along a single diameter. This corresponds to the evaluation of

$$
D=\frac{D_{1}+D_{2}}{2}
$$

In the present case, the contribution of lens aberrations to the distortion has been assumed zero, so if $D$ is not zero, the departure therefrom is a consequence of the camera tipping alone. It is evident that for small values of $\epsilon$

$$
\frac{D_{1}+D_{2}}{2}=\frac{f \epsilon^{2} \tan \beta}{\cos ^{2} \beta}
$$

Values of $D$ have been computed for selected values of $\epsilon$ and are shown in table 2. It is readily apparent that camera tipping does induce a resultant distortion that is not eliminated by the averaging process. This error in distortion produced by camera tipping can be safely neglected for values of $\epsilon$ less than 10 min but may assume serious proportions for values of $\epsilon$ greater than $20 \mathrm{~min}$. 
TAPLE 2. Distortion induced in a normally distortion-free lens by tipping the camera, equipped with a lens having an equivalent focal length of $150 \mathrm{~mm}$, through a small angle $\epsilon$

\begin{tabular}{|c|c|c|c|c|}
\hline \multirow{2}{*}{$\beta$} & \multicolumn{4}{|c|}{ Distortion for $\epsilon$ of -} \\
\hline & $10 \mathrm{~min}$ & $20 \mathrm{~min}$ & $30 \mathrm{~min}$ & $1^{\circ}$ \\
\hline $\begin{array}{l}\text { deg } \\
7.5 \\
15 \\
22.5 \\
30 \\
37.5 \\
45\end{array}$ & $\begin{array}{c}m m \\
0.000 \\
.000 \\
.001 \\
.001 \\
.002 \\
.003\end{array}$ & $\begin{array}{c}m m \\
0.001 \\
.002 \\
.002 \\
.004 \\
.006 \\
.010\end{array}$ & $\begin{array}{c}m m \\
0.002 \\
.003 \\
.006 \\
.009 \\
.014 \\
.023\end{array}$ & $\begin{array}{c}m m \\
0.006 \\
.013 \\
.022 \\
.035 \\
.056 \\
.091\end{array}$ \\
\hline
\end{tabular}

For the case of a real lens having measurable inherent distortion, it may be inferred that the distortion at a given angle $\beta$ is given reasonably closely by eq (18) provided that $\epsilon$ is kept less than $15 \mathrm{~min}$. If one is dealing with a lens which is required to show less than $0 \pm .020 \mathrm{~mm}$ of distortion referred to the equivalent focal length, it is possible to induce an error of $\pm 0.003 \mathrm{~mm}$ in the distortion referred to the calibrated focal length if $\epsilon$ is as great as $30 \mathrm{~min}$. It is also likely that small additional errors may arise in averaging for those values of $\beta$ where the distortion characteristics of the lens are changing rapidly with $\beta$. Even in such instances, the errors in distortion will usually be small compared with the total distortion.

\section{b. Evaluation of the Angle of Camera Tipping}

When a camera is tested under conditions such that the test targets are symmetrically located about the central target, it is possible to evaluate $\epsilon$, the angle of camera tipping, in a very simple manner. It can be shown for small values of $\epsilon$ that

$$
\frac{D_{1}-D_{2}}{2}=-f \epsilon \tan ^{2} \beta
$$

When $D_{1}, D_{2}, f$, and $\beta$ are known, $\epsilon$ can be computed from the relation

$$
\epsilon=\frac{\left(D_{1}-D_{2}\right)}{2 f \tan ^{2} \beta} .
$$

This expression is, of course, computed for the case of a distortion-free lens but can be used quite satisfactorily for a lens having distortion arising fron lens aberrations. This apparent anomaly results from the fact that the lens aberration is symmetrical about the axis and has the same value at $+\beta$ and at $-\beta$. Consequently, when the difference, $D_{1}-D_{2}$, is formed, the contribution to this difference from lens aberration is negligible, the only real contribution being the difference in lens distortion between the angles $\beta+\epsilon$ and $\beta-\epsilon$, which is usually very small for small values of $\epsilon$. This difference can become appreciable in regions where the distortion is changing rapidly with angle. In such cases, a correction can be made, if one knows the usual values of the distortion for the given type of lens, which usually will be satisfactory. Table 1 lists the value of $\left(D_{2}-D_{1}\right) / 2$ for one case of camera tipping. If $\epsilon$ is evaluated from the values listed, it will be found that the above approximation (eq (21)) yields the correct value of $\epsilon$.

The value of the equivalent focal length, $f$, used in eq (21) is the approximate value determined with the aid of eq (9). The departure of this value of $f$ from its corrected value will usually be too small to affect significantly the accuracy of the determination of $\epsilon$. However, the value of $\epsilon$, derived from eq (21), is sufficiently accurate for use in eq (8) to determine the correct value of the equivalent focal length $f$.

\subsection{Point of Symmetry}

It is clear from the foregoing discussion that if a camera is not properly alined for test and if computations of equivalent focal length and distortion are nonetheless performed in the usual manner, marked asymmetries in the distortion pattern will appear. The magnitude of the asymmetry will, of course, depend upon the amount the camera is tipped from the condition of proper alinement. In the field calibration method when the camera is not initially so alined that the focal plane is normal to the line connecting the front nodal point of the lens and the center target, an appreciable amount of asymmetric distortion is introduced. Methods have been developed that tend to reduce the magnitude of the asymmetric distortion. This usually involves locating a new point in the image plane with respect to which the distortion pattern becomes more nearly symmetrical. This point is called the "point of symmetry" $[6,11]$.

In the absence of prism effect, point $O$ in figure 1 is the principal point of the camera, and the distance $O^{\prime} O^{\prime \prime}$ is the shift of the central target image from the principal point. As $O^{\prime} O^{\prime \prime}=f \tan \epsilon$, and as the value of $f \tan \epsilon$ can be deduced from the measured value of the asymmetric distortion for a given value of $\beta$, the location of the principal point can be determined. For this condition the principal point and point of symmetry are identical.

When both prism effect and camera tipping are present, the value of $f \tan \epsilon$ gives the location of the point of symmetry for a selected value of $\beta$. For these conditions, the principal point and the point of symmetry do not coincide.

\section{Experimental Verification for Tipped Camera}

In the calibration of precision cameras by the field method, it is usual to locate a point about which the distortion is symmetrical. This point is called the point of symmetry [6] and this point is sometimes set into coincidence with the center of collimation instead of setting the principal point into coincidence with the center of collimation. The prevailing practice at most calibrating laboratories is to set either the principal point or the principal point of autocollimation [2] into coincidence with the center of collimation (or fiducial center). The principal point is located at the foot of the perpendicular dropped from the center of the exit pupil for the paraxial rays 
to the image plane and is the point customarily brought into coincidence with the center of collimation by the Bureau. The principal point of autocollimation is the image of an infinitely distant point located in the object space on a line perpendicular to the focal plane of the camera. This point is identical to the point referred to as the center cross in calibration made by the Bureau. In the absence of prism effect, the point of symmetry, principal point, and the center cross should coincide. The truth of the foregoing assertion is readily proved experimentally and the balance of this section is devoted to a simple experimental proof.

\subsection{Experimental Arrangement}

The camera selected for test was one which on calibration has been found to have negligible prism effect. The separation of the principal point and the center of collimation was less than $0.010 \mathrm{~mm}$; and the separation of the center cross and the principal point was too small to be measured. This camera was placed on the camera calibrator and tipped about a horizontal axis lying in a plane defined by the optical axes of the collimator banks III and IV (see figures 2 and 3 for diagrammatic sketches of the trace of this plane in the focal plane of the camera). To achieve a measured amount of camera tipping, a small prism was mounted in front of the autocollimating telescope, and the telescope alined to point at the center of the reticle in the central collimator of the camera calibrator. The prism was then removed. The camera under study was then

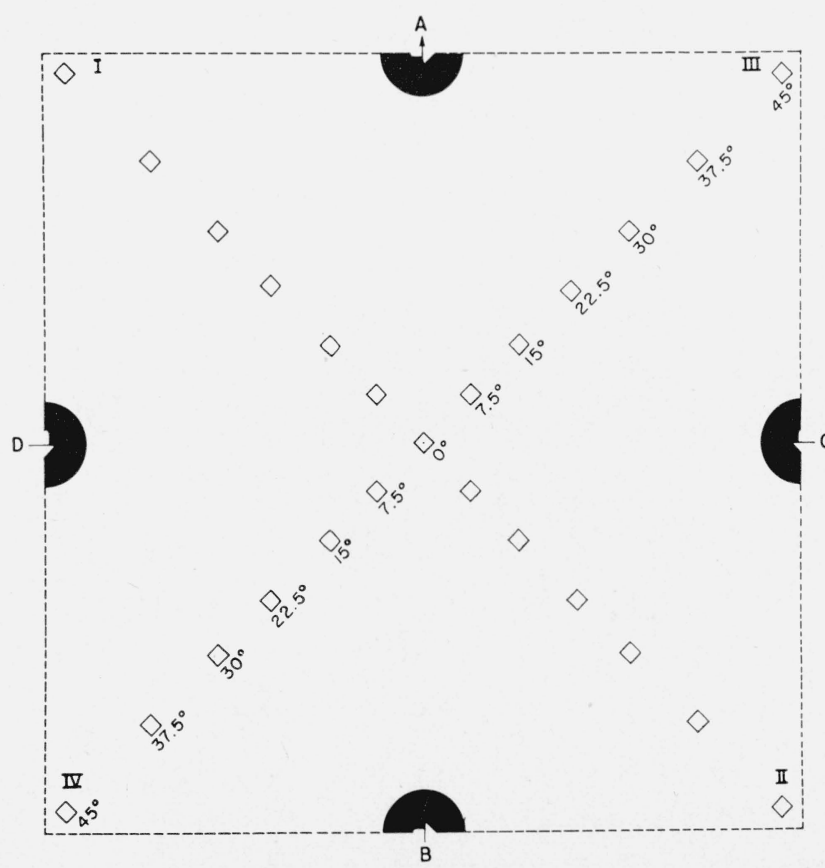

FIGURE 2. Schematic drawing of test negative obtained on camera calibrator showing the position of the collimation markers $A, B, C$, and $D$ and the images of the various collimator targets.

The four radial banks are indicated by the numerals I, II, III, and IV. This figure is to be considered in connection with plate $2 \mathrm{~B}$, figure 4 . placed in position on the calibrator. An optical plane parallel was set on the focal plane and the camera was tipped until its focal plane was normal to the optical axis of the autocollimating telescope. The measured deviation of the line of sight produced by the prism was $0.2583^{\circ}$. The tip of approximately 15 min accordingly may be expected to produce its maximum effect along the diagonal that is the intersection of the focal plane and the plane defined by the optical axes of the collimators in banks I and II.

In making the first negative (hereafter referred to as plate $1 \mathrm{~A}$ ), the camera was so oriented in azimuth, that marker A (which designates the line of flight) lay between the radial rows of images formed by the camera lens of the targets contained in the collimators of banks II and IV. Because of the tipping, all of the collimation markers are moved with respect to the target images toward quadrant I. Accordingly when the location of the central image is determined with respect to the center of collimation, $\mathrm{O}$, it will be displaced from the center of collimation along the diagonal toward the corner of quadrant II. The amount of this tip was measured directly and found to be approximately $0.2583^{\circ}$. In making the second, or check, negative (plate 2B), the camera is rotated $180^{\circ}$ about the optic axis of the lens with all other conditions remaining unchanged. Accordingly for plate $1 \mathrm{~A}$, the central image should appear in the quadrant $A O D$, and displaced from $O$ in direction II, while for plate $2 \mathrm{~B}$, the central image should appear in the quadrant $B O C$ and displaced from $O$ in the direction II. In the $180^{\circ}$ rotation the direction II remains unchanged and $A O D$ is replaced by $B O C$.
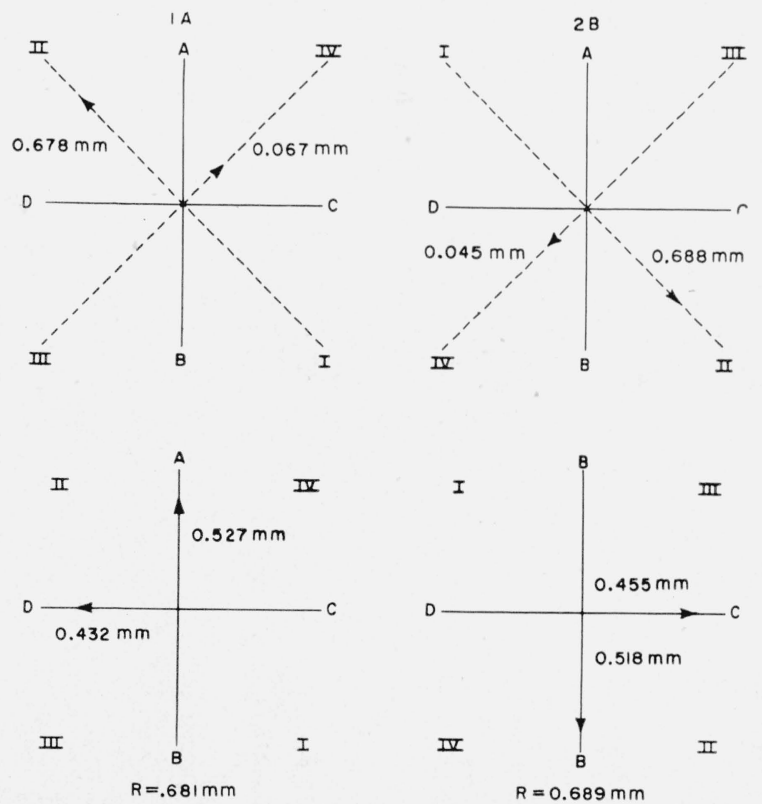

FiguRE 3. Displacement of the center image from the center of collimation produced by a camera tipping of approximately $15 \mathrm{~min}$.

The upper sketches show the displacements computed from the distortion measurements. The lower sketches show the displacement resolved along the coordinate axes defined by the collimation index markers. 


\subsection{Results of Measurement}

The distances separating all images on the negative from the image of the central collimator target were measured and are listed in tables 3 and 4 under the heading $r$. (The image of the central collimator target corresponds to $O^{\prime \prime}$ in figure 1 , and the values of $r$ are the measured values of $O^{\prime \prime} X^{\prime}$ and $O^{\prime \prime} Y^{\prime}$ for a series of values of $\beta$.) The symbols at the top and bottom of the columns headed $f \tan \beta$ indicate the orientation of the row of images with respect to the collimator banks and the fiducial markers in the focal plane (see figs. 2 and 3). The values of equivalent focal length were determined with the aid of eq (9) using the measured values of $\beta$, and the value of $f$ for each diameter appears at the top of the

TARLE 3. Measured values of distances separating images at angle $\beta$ from the center image for plane $1 \mathrm{~A}$

Camera tipped by approximately $15 \mathrm{~min}$ (deviation of normal by 0.5 prism diopter). $D$ is the distortion for each angle.

\begin{tabular}{|c|c|c|c|c|c|c|}
\hline \multirow[t]{2}{*}{$\beta$} & \multicolumn{3}{|c|}{$\begin{array}{c}f=153.368 \mathrm{~mm} \\
\mathrm{C}_{\mathrm{I}} \mathrm{B}\end{array}$} & \multicolumn{3}{|c|}{$\begin{array}{c}f=153.359 \mathrm{~mm} \\
{ }_{\text {III }} \text { D }\end{array}$} \\
\hline & $r$ & $f \tan \beta$ & $D$ & $r$ & $f \tan \beta$ & $D$ \\
\hline $\begin{array}{l}\text { deg } \\
45 \\
37.5 \\
30 \\
22.5 \\
15 \\
7.5 \\
0 \\
7.5 \\
15 \\
22.5 \\
30 \\
37.5 \\
45\end{array}$ & $\begin{array}{c}m m \\
152.368 \\
117.234 \\
88.313 \\
63.389 \\
41.023 \\
20.168 \\
0 \\
20.184 \\
41.121 \\
63.633 \\
88.775 \\
118.029 \\
153.690\end{array}$ & $\begin{array}{c}m m \\
153.197 \\
117.562 \\
88.464 \\
63.467 \\
41.062 \\
20.184 \\
0 \\
20.168 \\
41.061 \\
63.474 \\
88.467 \\
117.563 \\
153.205\end{array}$ & $\begin{array}{c}m m \\
-0.829 \\
-.328 \\
-.151 \\
-.078 \\
-.039 \\
-.016 \\
0 \\
0.016 \\
.060 \\
.159 \\
.308 \\
.466 \\
.485\end{array}$ & $\begin{array}{c}m m \\
153.259 \\
117.784 \\
88.653 \\
63.585 \\
41.126 \\
20.203 \\
0 \\
20.204 \\
41.127 \\
63.609 \\
88.695 \\
117.848 \\
153.378\end{array}$ & $\begin{array}{c}m m \\
153.508 \\
117.785 \\
88.612 \\
63.572 \\
41.126 \\
20.202 \\
0 \\
20.205 \\
41.108 \\
63.563 \\
88.616 \\
117.778 \\
153.512\end{array}$ & $\begin{array}{r}m m \\
-0.249 \\
-.001 \\
.041 \\
.013 \\
.000 \\
.001 \\
0 \\
-0.001 \\
.019 \\
.046 \\
.079 \\
.070 \\
-.134\end{array}$ \\
\hline $\begin{array}{l}37.5 \\
45\end{array}$ & \multicolumn{3}{|c|}{$\mathrm{A}_{\text {II }} \mathrm{D}$} & \multicolumn{3}{|c|}{$\mathrm{C}_{\text {IV }}{ }^{\mathrm{A}}$} \\
\hline
\end{tabular}

TAPLE 4. Measured values of distances separating images at angle $\beta$ from the center image for plate $2 B$

Conditions identical with those for $1 \mathrm{~A}$ except camera is rotated $180^{\circ}$ about its eptical axis.

\begin{tabular}{|c|c|c|c|c|c|c|}
\hline \multirow[t]{2}{*}{$\beta$} & \multicolumn{3}{|c|}{$\begin{array}{c}f=153.341 \mathrm{~mm} \\
\mathrm{D}_{\mathrm{I}} \mathrm{A}\end{array}$} & \multicolumn{3}{|c|}{$\begin{array}{c}f=153.356 \mathrm{~mm} \\
{ }_{\text {III }}{ }^{\mathrm{C}}\end{array}$} \\
\hline & $r$ & $f \tan \beta$ & $D$ & $r$ & $f \tan \beta$ & $D$ \\
\hline $\begin{array}{l}\text { deg } \\
45 \\
37.5 \\
30 \\
22.5 \\
15 \\
7.5 \\
0 \\
7.5 \\
15 \\
22.5 \\
30 \\
37.5 \\
45\end{array}$ & $\begin{array}{c}m m \\
152.346 \\
117.222 \\
88.299 \\
63.386 \\
41.020 \\
20.164 \\
0 \\
20.181 \\
41.121 \\
63.630 \\
88.769 \\
118.026 \\
153.701\end{array}$ & $\begin{array}{r}m m \\
153.170 \\
117.542 \\
88.449 \\
63.456 \\
41.055 \\
20.181 \\
0 \\
20.164 \\
41.054 \\
63.463 \\
88.452 \\
117.543 \\
153.178\end{array}$ & $\begin{array}{c}m m \\
-0.824 \\
-.320 \\
-.150 \\
-.070 \\
-.035 \\
-.017 \\
0 \\
0.017 \\
.067 \\
.167 \\
.317 \\
.483 \\
.523\end{array}$ & $\begin{array}{c}m m \\
153.280 \\
117.793 \\
88.655 \\
63.586 \\
41.127 \\
20.204 \\
0 \\
20.202 \\
41.120 \\
63.601 \\
88.682 \\
117.829 \\
153.356\end{array}$ & $\begin{array}{c}m m \\
153.504 \\
117.782 \\
88.610 \\
63.570 \\
41.125 \\
20.202 \\
0 \\
20.204 \\
41.107 \\
63.561 \\
88.614 \\
117.775 \\
153.508\end{array}$ & $\begin{array}{r}m m \\
-0.224 \\
.011 \\
.045 \\
.016 \\
.002 \\
.002 \\
0 \\
-0.002 \\
.013 \\
.040 \\
.053 \\
.054 \\
-.152\end{array}$ \\
\hline & \multicolumn{3}{|c|}{$\mathrm{B}_{\text {II }}{ }^{\mathrm{C}}$} & \multicolumn{3}{|c|}{${ }_{\text {IV }}^{B}$} \\
\hline
\end{tabular}

proper column. By using the proper $f$ for each set of measurements, the values of $f \tan \beta$ are computed, and the value of the distortion, $D$, obtained from the relation

$$
D=r-f \tan \beta .
$$

These values are shown under the headings $f$ tan $\beta$ and $D$. It is clear from the measurements that the principal effect has been produced along the directions I and II as planned. The asymmetric distortion appearing along the diameter I and II is shown graphically as curve 1 in figure 5 . It may be noted that $D_{\text {I }}$ (hereafter referred to as I) and $D_{\text {II }}$ (hereafter designated II), although opposite in sign, are of unequal magnitudes. This difference in magnitude is, in large part, a consequence of the large value of distortion inherent in the lens itself.

\section{a. Evaluation of the Angle of Camera Tipping}

Tables 5 and 6 show the evaluation of the angle of camera tipping $\epsilon$ from the distortion arising from the camera tipping. Values of $f \tan \epsilon$ are computed from the relation

$$
f \tan \epsilon=\frac{D_{2}-D_{1}}{2 \tan ^{2} \beta}
$$

derived in section 2.2 (b). For convenience in computation, the average values of $\tan \beta$ and $\tan ^{2} \beta$ for the actual measured angles are listed in table 7 . The values of $f \tan \epsilon$ are quite constant for the larger angles of $\beta$ for any one set of computations. For plate $1 \mathrm{~A}$ the values range from $0.658 \mathrm{~mm}$ at $45^{\circ}$ to $0.691 \mathrm{~mm}$ at $30^{\circ}$, with the values of $22.5^{\circ}$ and $37.5^{\circ}$ falling within this range. The simple average for these four values is $0.678 \mathrm{~mm}$, with the maximum departure being -0.020 at $45^{\circ}$ for a deviation of approximately 3 percent. This spread of values can be appreciably reduced if one corrects for the presence of distortion, which in this case would raise the value of $f \tan \epsilon$ at $45^{\circ}$ by 0.018 to 0.676 while producing changes not exceeding $\pm 0.002 \mathrm{~mm}$ for any of the other three values. Although this would substantially reduce the spread, this correction changes the average by less than 1 percent; it does not therefore appear worthwhile to include it in the present discussion. The values of $f \tan \epsilon$ at the smaller values of $\beta$ are less reliable, as a small error in $r$ produces a large error in $f \tan \epsilon$; for example, an error of $\pm 0.001 \mathrm{~mm}$ in $r$ at $\beta=7.5^{\circ}$ produces an error in $f \tan \epsilon$ of $\pm 0.029 \mathrm{~mm}$. The values of $f$ $\tan \epsilon$ at $7.5^{\circ}$ and $15^{\circ}$ accordingly are not used in evaluating the average value of $f \tan \epsilon$. This type of error decreases rapidly with increasing $\beta$ and becomes negligible for angles greater than $30^{\circ}$. Some of the small discrepancies at the larger angles doubtless arise as mentioned from the small differences in lens distortion at $\beta+\epsilon$ and $\beta-\epsilon$ which are small but noticeable for large $\epsilon$. These errors are, however, small in comparison to the larger values of $f \tan \epsilon$. 
TABLE 5. Computation of $f$ tan $\epsilon$ or displacement of the center image induced by camera tipping from distortion values in Table 3 for plate $1 \mathrm{~A}$

\begin{tabular}{|c|c|c|c|c|}
\hline$\beta$ & I & II & $\frac{\mathrm{II}-\mathrm{I}}{2}$ & $f \tan \epsilon$ \\
\hline $\begin{array}{l}\text { deg } \\
45 \\
37.5 \\
30 \\
22.5 \\
15 \\
7.5\end{array}$ & $\begin{array}{c}m m \\
-0.829 \\
-.328 \\
-.151 \\
-.078 \\
-.039 \\
-.016\end{array}$ & $\begin{array}{r}m m \\
0.485 \\
.466 \\
.308 \\
.159 \\
.060 \\
.016\end{array}$ & $\begin{array}{c}m m \\
0.657 \\
.397 \\
.230 \\
.118 \\
.050 \\
.016\end{array}$ & $\begin{array}{c}m m \\
0.658 \\
.676 \\
.691 \\
.688 \\
.698 \\
.924\end{array}$ \\
\hline \multicolumn{5}{|c|}{$\begin{array}{c}\text { Average }\left(22.5^{\circ} \text { to } 45^{\circ}\right) f \tan \epsilon=0.678 \mathrm{~mm}, \tan \epsilon= \\
0.004421, \text { and } \epsilon=0.2533^{\circ} .\end{array}$} \\
\hline$\beta$ & III & IV & $\frac{\mathrm{IV}-\mathrm{III}}{2}$ & $f \tan$ \\
\hline $\begin{array}{l}\operatorname{deg} \\
45 \\
37.5 \\
30 \\
22.5 \\
15 \\
7.5\end{array}$ & $\begin{array}{c}m m \\
-0.249 \\
-.001 \\
.041 \\
.013 \\
.000 \\
.001\end{array}$ & $\begin{array}{r}m m \\
-0.134 \\
.070 \\
.079 \\
.046 \\
.019 \\
-.001\end{array}$ & $\begin{array}{r}m m \\
0.058 \\
.036 \\
.019 \\
.016 \\
.008 \\
-.001\end{array}$ & $\begin{array}{l}m m \\
0.058 \\
.061 \\
.057 \\
.093 \\
.111 \\
-.058\end{array}$ \\
\hline
\end{tabular}

A verage $\left(22.5^{\circ}\right.$ to $\left.45^{\circ}\right) f \tan \epsilon=0.067 \mathrm{~mm}, \tan \epsilon=$ 0.000437 , and $\epsilon=0.0250^{\circ}$

Resultant $f \tan \epsilon=0.681 \mathrm{~mm}$, resultant $\tan \epsilon=$ 0.004440 , and $\epsilon=0.2544^{\circ}$

TABLE 6. Computation of $f$ tan $\epsilon$ or displacement of the center image induced by camera tipping from the distortion values in table 4 for plate $2 B$

\begin{tabular}{|c|c|c|c|c|}
\hline$\beta$ & I & II & $\frac{\mathrm{II}-\mathrm{I}}{2}$ & jtan. \\
\hline $\begin{array}{l}\mathrm{deg} \\
45 \\
37.5 \\
30 \\
22.5 \\
15 \\
7.5\end{array}$ & $\begin{array}{l}m m \\
-0.824 \\
-.320 \\
-.150 \\
-.070 \\
-.035 \\
-.017\end{array}$ & $\begin{array}{c}m m \\
0.523 \\
.483 \\
.317 \\
.167 \\
.067 \\
.017\end{array}$ & $\begin{array}{c}m m \\
0.674 \\
.492 \\
.234 \\
.118 \\
.051 \\
.017\end{array}$ & $\begin{array}{r}m m \\
0.675 \\
.684 \\
.703 \\
.689 \\
.711 \\
.982\end{array}$ \\
\hline \multicolumn{5}{|c|}{$\begin{array}{c}\left.\text { A verage }\left(22.5^{\circ} \text { to } 45^{\circ}\right) f \tan \epsilon=1\right) .688 \mathrm{~mm}, \tan \epsilon= \\
0.004486 \text {, and } \epsilon=0.2570^{\circ} .\end{array}$} \\
\hline$\beta$ & III & IV & $\frac{\mathrm{IV}-\mathrm{III}}{2}$ & $f \tan \epsilon$ \\
\hline $\begin{array}{l}\mathrm{deg} \\
45 \\
37.5 \\
30 \\
22.5 \\
15 \\
7.5\end{array}$ & $\begin{array}{r}m m \\
-0.224 \\
.011 \\
.045 \\
.016 \\
.002 \\
.002\end{array}$ & $\begin{array}{r}m m \\
-0.152 \\
.054 \\
.068 \\
.040 \\
.013 \\
-.002\end{array}$ & $\begin{array}{c}m m \\
0.036 \\
.022 \\
.012 \\
.012 \\
.006 \\
-.002\end{array}$ & $\begin{array}{l}m m \\
0.036 \\
.037 \\
.036 \\
.070 \\
.083 \\
-.115\end{array}$ \\
\hline \multicolumn{5}{|c|}{$\begin{array}{c}\text { Average }\left(22.5^{\circ} \text { to } 45^{\circ}\right) f \tan \epsilon=0.045 \mathrm{~mm}, \tan \epsilon= \\
0.000293, \text { and } \epsilon=0.0168^{\circ} \text {. } \\
\text { Resultant } f \tan \epsilon=0.689, \text { resultant tan } \epsilon=0.004492, \\
\text { and } \epsilon=0.2574^{\circ} \text {. }\end{array}$} \\
\hline
\end{tabular}

Although care was taken to ensure that the maximum effect of camera tipping would take place along diagonals I and II, the setting in azimuth was not quite perfect so a small effect of the tip manifested itself in directions III and IV. The computation of this component of $f \tan \epsilon$ is shown in the lower portions of tables 5 and 6 . The full magnitude of $f \tan \epsilon$ is then obtained by finding the square root of the sum of the squares of the two orthogonal components. The resultant $f \tan \epsilon$ for plate $1 \mathrm{~A}$ is 0.681 , and the corresponding value for plate $2 \mathrm{~B}$ is
TABLE 7. Values of average tangent and tangent squared for use in interpreting data from camera calibrator

The measured values of $\boldsymbol{\beta}$ are used in computing $\tan \boldsymbol{\beta}$.

\begin{tabular}{|c|c|c|}
\hline$\beta$ & $\tan \beta$ & $\tan { }^{2} \boldsymbol{\beta}$ \\
\hline \multicolumn{3}{|c|}{ COLLIMATOR BANKS I AND II } \\
\hline $\begin{array}{l}\text { deg } \\
7.5 \\
15 \\
22.5 \\
30 \\
37.5 \\
45\end{array}$ & $\begin{array}{r}0.1315530 \\
.2677322 \\
.4138446 \\
.5768210 \\
.7665412 \\
.9989116\end{array}$ & $\begin{array}{r}0.0173062 \\
.0716805 \\
.1712674 \\
.3327225 \\
.5875854 \\
.9978244\end{array}$ \\
\hline \multicolumn{3}{|c|}{ COLLIMATOR BANKS III AND IV } \\
\hline $\begin{array}{l}7.5 \\
15 \\
22.5 \\
30 \\
37.5 \\
45\end{array}$ & $\begin{array}{r}0.1317395 \\
.2681092 \\
.4144998 \\
.5778216 \\
.7680095 \\
1.0009800\end{array}$ & $\begin{array}{r}0.0173553 \\
.0718825 \\
.1718101 \\
.3338778 \\
.5898386 \\
1.0019610\end{array}$ \\
\hline
\end{tabular}

0.689. It is evident that excellent agreement exists between the values, even though the camera was rotated $180^{\circ}$ and the direction of the effect thereby reversed.

The values of $f \tan \epsilon$ computed from the measurements of plates $1 \mathrm{~A}$ and $2 \mathrm{~B}$ are the amounts by which the center image has been displaced from the position it occupies when the camera under test has been properly oriented by autocollimation. As the center image was initially in near coincidence with the center of collimation, the computed displacement, $f \tan \epsilon$, can be regarded as a displacement with respect to the center of collimation. The magnitudes and directions of $f$ tan $\epsilon$ derived from tables 5 and 6 are shown in the upper part of figure 4 for plates $1 \mathrm{~A}$ and $2 \mathrm{~B}$. In the lower part of the figure, the displacements, originally determined along the diagonals, have been resolved into their components along $A B$ and $C D$, which form the reference system afforded by lines connecting opposite pairs of collimation index markers. The resultant, $R$, for each plate is shown at the bottom. It is clear that there is remarkably good agreement between the values of $R$ for plates $1 \mathrm{~A}$ and $2 \mathrm{~B}$.

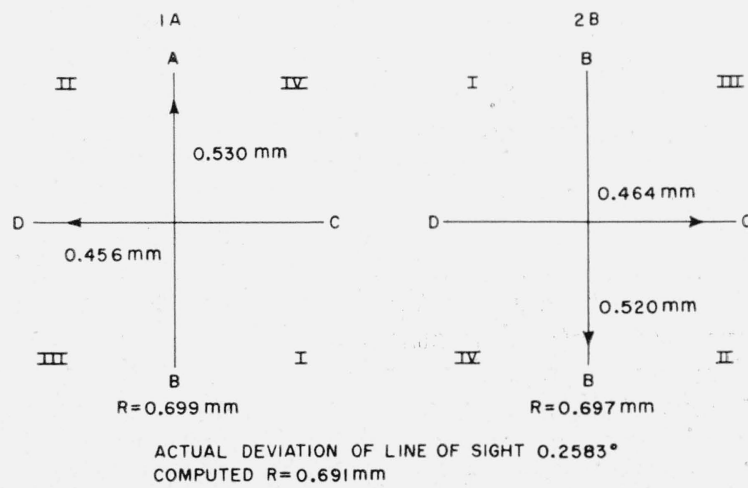

Figure 4. Measured displacement of the center image from the center of collimation on plates $1 A$ and $2 B$.

The computed $R$ is the resultant displacement expected for the measured amount of camera tipping introduced. 
Finally, the positions of the displaced center image were measured on the plates themselves with respect to the center of collimation. The results of these measurements are shown in figure 4. Also shown in the lower part of figure 4 is the actual measured value of the induced angle of camera iipping, which is $0.2583^{\circ}$, and the computed value of $f \tan \epsilon$ derived from this value of $\epsilon$. Comparison of these results with those shown in figure 3 demonstrates that the displacements of the center image computed from the asymmetrical values of the distortion agree in direction and magnitude with the observed shift that actually took place. The small discrepancies that remain may be attributed to several factors that include the presence of a small residual prism in the lens, small additional distortions induced by tipping not completely compensated, small amounts of plate curvature, and small errors in $r$ and $\beta$.

Accordingly the resultant $f \tan \epsilon$ has been determined in three ways with the following results: (1) $f$ tan $\epsilon$ has been computed from the measured values of the asymmetric distortion, and its average value for plates $1 \mathrm{~A}$ and $2 \mathrm{~B}$ is $0.685 \mathrm{~mm}$; (2) $f$ tan $\epsilon$ has been measured directly on the negative with respect to the center of collimation, and its average value for plates $1 \mathrm{~A}$ and $2 \mathrm{~B}$ is 0.698 ; (3) finally, $f$ tan $\epsilon$ has been computed on the basis of the known value of the induced camera tipping, and its value is 0.691 . In view of the relatively large value of $f$ tan $\epsilon$, it is clear that excellent agreement exists among the three methods. This accordingly justifies the use of eq (23) in evaluating the angle of camera tipping from the asymmetric values of distortion and provides a simple method of locating the point of symmetry on the basis of distortion measurements.

\section{b. Evaluation of the Distortion}

In point of symmetry operations, it is customary to equalize the distortions. This can be done in the present case by subtracting the distortion produced by tipping from the measured values. This is done in table 8 for one diagonal of plate $1 \mathrm{~A}$. In table 8 , column 1 lists the values of the measured asymmetric distortion for one diagonal, and column 2 lists the average asymmetric distortion computed for $f$ tan $\epsilon=0.678 \mathrm{~mm}$. By adding the values in 2 to those in 1 , the resultant lens distortion is obtained, which is shown in column 3. The distortion values in 3 are nearly equal for corresponding values of $\beta$. This process is shown graphically in figure 5 . In the figure, curve 1 shows the values of the distortion computed with the displaced central image as the center. Curve 2 shows the amount of this distortion attributable to camera tipping. Curve 3 shows the amount of distortion still remaining after the correction for camera tipping is applied. It is evident that curve 3 is already quite symmetrical and closely approximates a typical distortion curve for this variety of wide-angle lens. In the table, column 4 shows the complete equalization obtained by averaging. Column 5 shows the difference between 3 and 4 and leads to the belief that complete equalization was not achieved because of the introduction
TABLE 8. Adjustment of distortion values from tipped camera data for plate $1 \mathrm{~A}$, collimator banks $I$ and II

$\mathrm{EFL}=153.368 \mathrm{~mm}, f \tan \epsilon=0.678 \mathrm{~mm}, \mathrm{CFL}=153.310 \mathrm{~mm}$.

\begin{tabular}{|c|c|c|c|c|c|c|c|}
\hline$\beta$ & $1 \mathrm{a}$ & 2 & 3 & 4 & 5 & 6 & 7 \\
\hline deg & $m m$ & $m m$ & $m m$ & $m m$ & $m m$ & $m m$ & $m m$ \\
\hline 45 & -0.829 & 0.678 & -0.153 & -0.172 & 0.019 & 0.058 & -0.114 \\
\hline 37.5 & -.328 & .398 & .070 & .069 & .001 & .044 & .113 \\
\hline 30 & -.151 & .226 & .075 & .078 & -.003 & .033 & .111 \\
\hline 22,5 & -.078 & .116 & .038 & .040 & $\begin{array}{r}.002 \\
-.002\end{array}$ & .024 & .064 \\
\hline 15 & -039 & .049 & .010 & .010 & .000 & .016 & .026 \\
\hline 7.5 & -.016 & .012 & -.004 & .000 & -.004 & .008 & .008 \\
\hline 0 & & 0 & 0 & 0 & 0 & 0 & 0 \\
\hline 7.5 & .016 & -0.012 & .004 & .000 & .004 & - . & -.... \\
\hline 15 & .060 & -.049 & .011 & .010 & .001 & ..... & $\ldots$ \\
\hline 22.5 & .159 & -.116 & .043 & .040 & .003 & - n & - \\
\hline 30 & .308 & -.226 & .082 & .078 & .004 & - & ....... \\
\hline 37.5 & .466 & -.398 & .068 & .069 & -.001 & -... & $\ldots$ \\
\hline 45 & .485 & -.676 & -.191 & -.172 & -.019 & $\ldots$ & $\ldots$ \\
\hline
\end{tabular}

a Column:

1. Initial asymmetric values of distortion.

2. $f \tan \in \tan ^{2} \beta$ (contribution from camera tipping).

3. Value of distortion compensated for camera tipping

4. Averaged values of distortion referred to equivalent focal length.

5 . Departure of compensated distortion from average.

6. $\Delta f \tan \beta$, where $\Delta f=0.058 \mathrm{~mm}$.

7. Values of distortion referred to calibrated focal length.

of a small amount of residual distortion induced by plate tipping. Column 6 shows the correction in distortion introduced by changing from the EFL to the CFL by amount $\Delta f=0.058 \mathrm{~mm}$. Column 7 shows the values of the distortion referred to the calibrated focal length. These final values of the distortion referred to the CFL are nearly the same as those obtained from the properly oriented camera; the small differences arising from the uncompensated distortion introduced by camera tipping, which in this instance would not exceed $\pm 0.003 \mathrm{~mm}$.

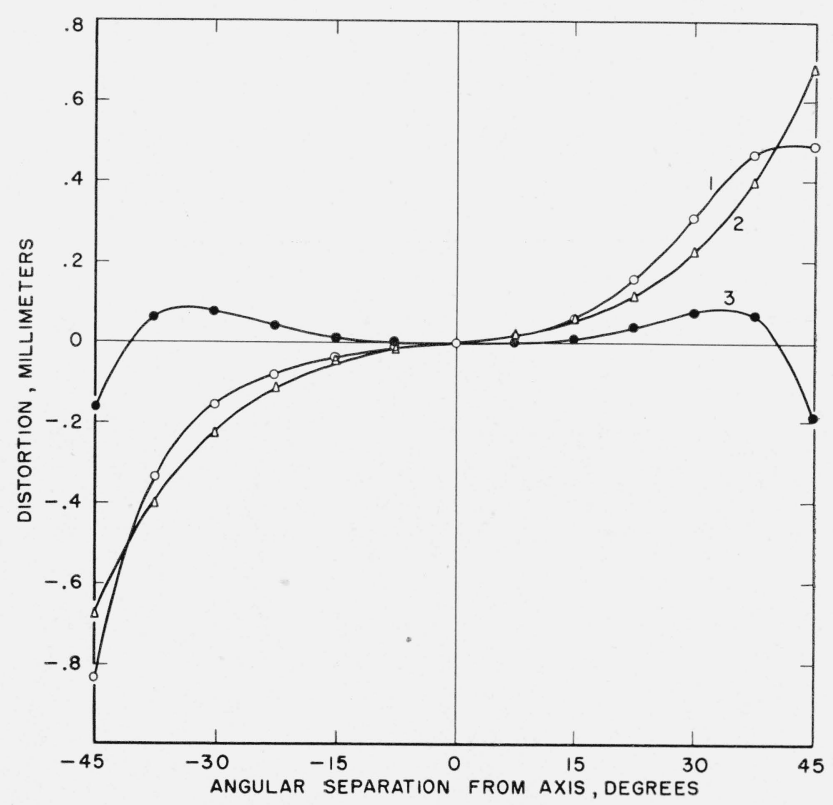

FiguRE 5. Evaluation of the true values of distortion from the asymmetric measured values.

The open circles (curve 1) show the actual values of distortion as initially determined from the measurements. The triangles (curve 2) show the values of asymmetric distortion produced by a tipping of amount $\epsilon=0.2533^{\circ}$ (see table 5) or $15.2 \mathrm{~min}$. The closed circles (curve 3 ) show the symmetrical distortion pattern remaining when curve 2 is subtracted from curve 1 . 


\section{Discussion}

When a camera under calibration is initially so alined that the focal plane of the camera is not normal to the line joining the front nodal point of the lens and the distant target, the values of the distortion based on the assumption that the central image is indeed in its proper position will be asymmetrical about this point. The values of the distortion at points located at equal but opposite angles from the center can be used to evaluate the magnitude and direction of the displacement of the central image from its position for the condition of true normality. On making this correction, which can be done in a simple manner, the point of symmetry can be readily located. For the case of no prism effect on the lens, the point so located is the point of autocollimation or center cross. Accordingly it is clear that for these conditions, the point of autocollimation and point of symmetry are identical and can be regarded as the true principal point of the camera.

The author expresses his appreciation to other members of the staff for assistance rendered during the course of this work. The negatives used in the confirmatory experiments were made by W. P. Tayman. Measurements on the negatives were made by W. R. Darling. The drawings were made by E. C. Watts.

\section{References}

[1] United States Department of Agriculture Specification No. A-APC-1102 (as approved March 1940).

[2] Report of International Commission I-Proposal for international photogrammetric lens tests, Photogrammetria III, 103 (1950-1951).

[3] R. H. Field, A device for locating the principal point markers of air cameras, The Canadian Surveyor, July 1949 (N. R. C. No. 1979).

[4] F. E. Washer, Locating the principal point of precision airplane mapping cameras, J. Research NBS 2\%, 405 (1941) RP1428.

[5] F. E. Washer and F. A. Case, Calibration of precision airplane mapping camera, Photogrammetric Engineering XVI, 502 (1950); J. Research NBS 45, 1 (1950) $\mathrm{RP} 2108$

[6] E. D. Sewell, Field calibration of aerial mapping cameras, Photogrammetric Engineering, XVI, 363, (1948); Manual of Photogrammetry by the American Society of Photogrammetry, 137-176 (1952).

[7] J. V. Sharp and H. H. Hayes, Effect on map production of distortions in photogrammetric systems, Photogrammetric Engineering XV, 159 (1949).

[8] J. V. Sharp, Increased accuracy of the multiplex system, Photogrammetric Engineering XV, 430 (1949).

[9] J. V. Sharp, Basic factors in photogrammetric instrument performance, Photogrammetric Engineering XVI, $118(1950)$

[10] P. D. Carman, Photogrammetric errors from camera lens decentering, J. Opt. Soc. Am. 39, 951 (1949).

[11] R. Roelofs, Distortion, principal point, point of symmetry and calibrated principal point, Photogrammetria II, 49 (1950-1951).

[12] W. Roos, Über die Definition des Bildhauptpunktes und der Aufnahmeachse, Allgem. Vermess Nachr. (Bildm. u. Luftbildwesen), p. 235 (1950)

[13] F. E. Washer, Sources of error in various methods of airplane camera calibration. Photogrammetric Engineering XXII (to be published in September 1956).

Washington, March 22, 1956. 